\title{
Characterization of ATP-binding Cassette Transporter Genes in Silkworm, Bombyx Mori
}

\author{
Fengpeng $\mathrm{Li}^{1}$, Xuefang Wang ${ }^{1}$, Ying $\mathrm{Xu}^{1}$, Jinmei $\mathrm{Wu}^{1,2, \text {, }}$ \\ ${ }^{1}$ College of Biotechnology, Jiangsu University of Science and Technology, Zhenjiang City, P. R. China \\ ${ }^{2}$ The Sericultural Research Institute, Chinese Academy of Agricultural Sciences, Zhenjiang City, P. R. China
}

Email address:

jwuus@hotmail.com (Jinmei Wu)

\section{To cite this article:}

Fengpeng Li, Xuefang Wang, Ying Xu, Jinmei Wu. Characterization of ATP-binding Cassette Transporter Genes in Silkworm, Bombyx Mori. American Journal of Bioscience and Bioengineering. Vol. 3, No. 5, 2015, pp. 123-133. doi: 10.11648/j.bio.20150305.27

\begin{abstract}
Background: ATP-binding cassette (ABC) transporters are transmembrane proteins that utilize the energy of adenosine triphosphate (ATP) binding and hydrolysis to transport various substrates across extra and intracellular membranes, including metabolic products, lipids and sterols, and drugs. They play important roles in various processes of life, especially in drug resistance, metabolism and development. Objective: Identify the ATP-binding cassette (ABC) transporter gene family and their members in the genome of silkworm, Bombyx mori. Method: Bioinformatics and phylogenetic analysis were used in the study. Results: We identified $47 \mathrm{ABC}$ proteins in the silkworm genome, which possesses members of all current $\mathrm{ABC}$ subfamilies $\mathrm{A}$ to $\mathrm{H}$. $\mathrm{ABC}$ proteins of silkworm were compared to those from worm, fruit fly and human. A high conservation of silkworm $\mathrm{ABC}$ transporters were observed for proteins involved in fundamental cellular processes, including the half transporters of the $\mathrm{ABCB}$ subfamily, which function in iron metabolism and transport of $\mathrm{Fe} / \mathrm{S}$ protein precursors, and the members of subfamilies $\mathrm{ABCD}, \mathrm{ABCE}$ and $\mathrm{ABCF}$, which have roles in very long chain fatty acid transport. Both $\mathrm{ABCE}$ and $\mathrm{F}$ gene products may be involved in an innate immune response to viral infections. As in the fly, $\mathrm{ABCH}$ proteins are inverse half-transporters showing the same domain architecture as the members of the ABCG subfamily, and ABCG transporters involve in transportation of ommochrome precursors and uric acid into pigment granules and urate granules. Conclusion: These results paved the way for further study on the function of the $\mathrm{ABC}$ transporters in silkworm, Bombyx mori.
\end{abstract}

Keywords: ATP-binding Cassette Transporters, Silkworm, ABCG, Cholesterol Efflux, Multidrug Resistance-associated Protein, Insect

\section{Introduction}

ATP-binding cassette $(\mathrm{ABC})$ proteins constitute one of the largest protein superfamilies and are present in all organisms from bacteria to human $[1,2,3,4]$. This protein family is characterized by an ATP-binding domain that binds and hydrolyses ATP to provide the energy to transport a variety of molecules against concentration gradients across biological membranes $[5,6]$. In addition to transporters, $\mathrm{ABC}$ proteins also comprise ion channels, regulators of ion channels, receptors, and proteins with roles in ribosome assembly and translation. This binding site is quite conserved throughout the biological kingdoms containing three motifs, the Walker A and B motifs that are typically about 100 amino acids apart plus a signature motif whose position can vary with respect to the other two. $\mathrm{ABC}$ proteins share conserved domain architecture. A functional transporter requires the cooperation of two transmembrane domains (TMD) and two cytosolic nucleotide binding domains (NBDs, also called ATP-binding cassettes). Eukaryotic $\mathrm{ABC}$ proteins are either full transporters combining all required domains in one polypeptide (two TMDs and two NBDs), or half-transporters consisting of one TMD and one NBD that need to form homo-or heterodimers to constitute a functional pump. According to their domain architecture and sequence, metazoan $\mathrm{ABC}$ transporters are divided into subfamilies, of which seven (A to G) exist in human [7]. Three deduced Drosophila genes encoded proteins with ATP-binding site sequences that cluster into a new branch on the calculated phylogenetic tree and this new subgroup was called $\mathrm{ABCH}$ [8] The $\mathrm{H}$ subfamily is missing in mammals, but has one member in zebrafish [9]. The human genome has 48 genes encoding $\mathrm{ABC}$ proteins, of which 17 have been linked to hereditary diseases, including cystic fibrosis, stargardt disease, 
adrenoleukodystrophy and disorders of cholesterol metabolism [10, 11, 12, 13]. As reported recently, ABC transporters may play an important role in cancers $[14,15,16]$ and in reproduction [17].

One of the most studied eukaryotic ABC transporters is probably the Drosophila melanogaster white gene. Mutations in this gene have provided a classical, selective eye color marker both for Drosophila as well as for a number of other organisms. Another major impetus for research on these fascinating proteins was the discovery by Victor Ling and his collaborators [18], that $\mathrm{ABC}$ transporters are associated with drug resistance in Chinese hamster ovary (CHO) cells, a discovery that was quickly extended to human cell lines and tumors $[11,12,14,15,16]$. This research led to the identification of new $\mathrm{ABC}$ transporter proteins associated with resistance to a variety of toxic compounds in a large number of organisms. The first eukaryotic $\mathrm{ABC}$ transporter discovered was the human (h) drug efflux transporter MDR (multidrug resistance) P-glycoprotein (hABCB1/MDR1), the name of which reflects that its expression in cancers can cause a decreased cellular drug accumulation (initially referred to as drug permeability, ' $\mathrm{P}$ '), resulting in the resistance of tumors against chemotherapy [20, 21]. Subsequent studies have identified further $\mathrm{ABC}$ proteins that are drug efflux pumps and can cause MDR in cancers, including the multidrug resistance associated protein (hABCC1/MRP1) [22] and the breast cancer related protein (hABCG2/BCRP) [23]. Drug efflux transporters are found in ABC subfamilies B, C and G [24], and in normal tissues often show an apical expression in epithelia involved in excretion or forming boundaries of the body, reflecting their role in the biochemical defense against toxicants [25]. While interest in $\mathrm{ABC}$ transporters may have started in Drosophila, little work has been done in this insect toward understanding the role of $\mathrm{ABC}$ transporters in cellular detoxification. The Drosophila genome project identified a number of new ABC transporter genes that do not have associated phenotypes. The physiological role of these transporters is not fully understood in Drosophila or in humans where there has been an extensive amount of research. As a first step toward understanding the function of these transporters in insects, we are identifying genes encoding these proteins in the recently released silkworm genome sequence. To unravel phylogenetic relationships, $\mathrm{ABC}$ proteins of silkworm were compared to those from worm, fruit fly and human. According to ABC proteins' function in human and fly, some homologous proteins' function was predicted in silkworm. As a primarily model insect of high economical importance, researching the silkworm $\mathrm{ABC}$ transporter proteins may be useful not only for revealing evolutionary relationship and function of the $\mathrm{ABC}$ transporter proteins in other organisms but also in the treatment of drug resistance in human diseases.

\section{Methods}

To identify putative ATP-binding cassette genes in the silkworm genome sequence, Tblastn searches were performed on silkworm genome sequence assembly [26] using the highly conserved NBD [27] of different Drosophila melanogaster $\mathrm{ABC}$ proteins as queries. One search was carried out per subfamily, each using the sequence of the NBD (as defined by interpro domain IPR003439) of a representative Drosophila protein (A:CG1718; B:CG3879(mdr49); C:CG9270; D:CG12703; E:CG5651; F: CG9330; G: white; H: CG9990).If the Drosophila transporter had two NBDs, the N-terminal domain was used. Hits from individual subfamily specific tblastn searches (E-value of 10-5) significantly overlapped, with each search retrieving loci of genes of the query and other subfamilies. Increasing the E-value to 10-4 increased the degree of overlap between individual searches, but had no effect on the total number of genes, suggesting that our search strategy gave an exhaustive representation of those sequences in the current version of the silkworm genome that show homology to ABC-transporter NBD domains. To assign putative silkworm $\mathrm{ABC}$ genes to $\mathrm{ABC}$ subfamilies, the NBDs of preliminary gene models were extracted using the ScanProsite facility [28] with predicted protein sequences and the prosite profile PS50893. NBDs were then subjected to a phylogenetic analysis together with those of fruit fly and human $\mathrm{ABC}$ transporters, using neighbour joining and bootstrapping with 2000 replicates in the program package phylip3.67.This allowed unequivocal assignment of silkworm transporters to ABC subfamilies. Gene models were then refined on the basis of EST support. The subfamily assignment of silkworm $\mathrm{ABC}$ proteins was confirmed by protein BLASTP analyses of the manually corrected models on the National Center for Biotechnology Information website. Separate phylogenetic analyses on silkworm, yeast, worm, fruitfly and human $\mathrm{ABC}$ transporters were then carried out every subfamily, using the same methodology as above on full sequences.

Table 1. Summary of the ATP-binding protein genes identified in the silkworm genome sequence.

\begin{tabular}{|c|c|c|c|c|c|c|c|c|}
\hline Sub - family & Protein name $^{a}$ & $\begin{array}{l}\text { Amino } \\
\text { acids }\end{array}$ & Location scaffold & $\begin{array}{l}\text { Orien - } \\
\text { tation }\end{array}$ & $\begin{array}{l}\text { Chromo - } \\
\text { some }\end{array}$ & Predicted topology & $\begin{array}{l}\text { EST } \\
\text { support }\end{array}$ & Comments \\
\hline \multirow[t]{6}{*}{ A } & $\mathrm{Bm} 012789$ & 1794 & $3058: 7588846-7625968$ & - & 16 & (7TM-NBD)2 & $\mathrm{Y}$ & 3 gap \\
\hline & $\mathrm{Bm} 007221$ & 1747 & $2873: 43128-67099$ & + & 17 & (8/4TM-NBD)2 & $\mathrm{Y}$ & 3 gap \\
\hline & $\mathrm{Bm} 004187$ & 452 & $2770: 824533-837264$ & + & 19 & 2TM-NBD & $\mathrm{N}$ & 3 gap \\
\hline & $\mathrm{Bm} 007217$ & 905 & $2873: 124005-140286$ & - & 17 & 3TM-NBD-3TM & $\mathrm{Y}$ & \\
\hline & $\mathrm{Bm} 007218$ & 416 & 2873:71197-79589 & - & 17 & TM-NBD & $\mathrm{Y}$ & \\
\hline & $\mathrm{Bm} 009503$ & 1040 & 2953:1661521-1682998 & + & 14 & 6TM-NBD & $\mathrm{Y}$ & 1 gap \\
\hline B & $\mathrm{Bm} 000725$ & 1149 & $1690: 6582863-6601286$ & + & 1 & (3/5TM-NBD)2 & $\mathrm{Y}$ & 1gap \\
\hline
\end{tabular}




\begin{tabular}{|c|c|c|c|c|c|c|c|c|}
\hline Sub - family & Protein name ${ }^{a}$ & $\begin{array}{l}\text { Amino } \\
\text { acids }\end{array}$ & Location scaffold & $\begin{array}{l}\text { Orien - } \\
\text { tation }\end{array}$ & $\begin{array}{l}\text { Chromo - } \\
\text { some }\end{array}$ & Predicted topology & $\begin{array}{l}\text { EST } \\
\text { support }\end{array}$ & Comments \\
\hline \multirow{23}{*}{$\mathrm{C}$} & $\mathrm{Bm} 000724$ & 1258 & $1690: 6553161-6573490$ & + & 1 & (4/6TM-NBD)2 & $\mathrm{Y}$ & \\
\hline & $\mathrm{Bm} 011228$ & 1311 & $3026: 3885423-3912497$ & _- & 23 & $(6 \mathrm{TM}-\mathrm{NBD}) 2$ & $\mathrm{Y}$ & 1gap \\
\hline & $\mathrm{Bm} 009452$ & 1312 & 2953:1966564-1939875 & _- & 14 & $(5 / 4 \mathrm{TM}-\mathrm{NBD}) 2$ & $\mathrm{~N}$ & 3gap \\
\hline & $\mathrm{Bm} 007494$ & 1268 & 2887:1965221-1945690 & _- & 15 & $(6 / 5 \mathrm{TM}-\mathrm{NBD}) 2$ & $\mathrm{Y}$ & 1gap \\
\hline & $\mathrm{Bm} 012743$ & 601 & $3056: 487542-500932$ & + & 22 & 3TM-NBD & Y & 5 gap \\
\hline & $\mathrm{Bm} 005473$ & 850 & 2828:3932738-3950945 & + & 8 & 10TM-NBD & $\mathrm{Y}$ & 1gap \\
\hline & Bm008523 & 994 & 2902:7389930-7412837 & + & 18 & 2TM-NBD & Y & 1gap \\
\hline & Bm004142 & 581 & $2767: 4285226-4299688$ & + & 19 & 2TM-NBD & $\mathrm{Y}$ & 2gap \\
\hline & $\mathrm{Bm} 007735$ & 1167 & 2888:1038895-1007371 & _- & 15 & (5/4TM-NBD)2 & Y & 5 gap \\
\hline & Bm003359 & 760 & $2655: 624399-606966$ & - & 15 & (2/1TM-NBD)2 & $\mathrm{N}$ & 3 gap \\
\hline & $\mathrm{Bm} 011220$ & 1263 & $3026: 4292000-4262367$ & - & 23 & (4/6TM-NBD)2 & $\mathrm{Y}$ & 3gap \\
\hline & Bm006882 & 1334 & 2859:1597393-1617896 & + & 10 & $(5 \mathrm{TM}-\mathrm{NBD}) 2$ & $\mathrm{Y}$ & 2gap \\
\hline & Bm010332 & 1180 & 2990:30206-12995 & _- & 12 & $(1 / 3 \mathrm{TM}-\mathrm{NBD}) 2$ & $\mathrm{Y}$ & 1 gap \\
\hline & $\mathrm{Bm} 010636$ & 1594 & 2998:322760-305046 & - & 12 & $(10 / 5 T M-N B D) 2$ & $\mathrm{~N}$ & \\
\hline & $\mathrm{Bm} 007738$ & 983 & 2888:930623-908517 & - & 15 & 7TM-NBD & $\mathrm{Y}$ & 3gap \\
\hline & Bm007793 & 305 & 2888:999476-1002597 & + & 15 & NBD & $\mathrm{N}$ & \\
\hline & $\mathrm{Bm} 007785$ & 728 & 2888:767551-792765 & + & 15 & 6TM-NBD & $\mathrm{Y}$ & 4gap \\
\hline & Bm007769 & 297 & 2888:134904-141029 & + & 15 & NBD & $\mathrm{Y}$ & 1gap \\
\hline & Bm007792 & 658 & 2888:984948-994967 & + & 15 & 5TM-NBD-TM & $\mathrm{N}$ & 1gap \\
\hline & $\mathrm{Bm} 007784$ & 540 & 2888:751792-764508 & + & 15 & 5TM-NBD & Y & \\
\hline & Bm010331 & 496 & 2990: 44300-39940 & _- & 12 & 2TM-NBD & $\mathrm{Y}$ & \\
\hline & Bm010330 & 1251 & 2990: $78780-44879$ & - & 12 & 11TM-NBD-2TM & $\mathrm{Y}$ & 1gap \\
\hline & Bm010849 & 779 & 3004: 61144-47746 & - & Not known & TM-NBD-5TM & $\mathrm{Y}$ & \\
\hline \multirow[t]{2}{*}{ D } & Bm004616 & 754 & 2801: 381693-398156 & - & 27 & NBD & $\mathrm{N}$ & 3gap \\
\hline & $\mathrm{Bm} 012688$ & 503 & $3055: 13802-26162$ & + & 22 & 2TM-NBD & $\mathrm{Y}$ & \\
\hline $\mathrm{E}$ & Bm010129 & 647 & 2986:3437097-3447398 & _- & 7 & NBD-NBD & $\mathrm{Y}$ & 1gap \\
\hline $\mathrm{F}$ & Bm007869 & 906 & $2888: 4038262-4052670$ & + & 15 & NBD-NBD & $\mathrm{Y}$ & 1gap \\
\hline \multirow{9}{*}{ G } & Bm002004 & 622 & $2210: 4610405-4619608$ & _- & 1 & NBD-NBD & $\mathrm{Y}$ & \\
\hline & Bm006964 & 638 & $2860: 2849092-2861275$ & + & 10 & NBD-NBD & $\mathrm{Y}$ & 1gap \\
\hline & Bm005094 & 727 & 2823:124639-134335 & _- & 25 & NBD-5TM & $\mathrm{N}$ & 1gap \\
\hline & Bm002922 & 664 & $2575: 3237764-3274876$ & + & 10 & NBD-6TM & $\mathrm{Y}$ & 7 gap \\
\hline & $\mathrm{Bm} 000220$ & 823 & 1681:5597989-5616076 & _- & 22 & NBD-6TM & $\mathrm{N}$ & 2gap \\
\hline & Bm012035 & 473 & $3034: 3590330-3612716$ & - & 11 & NBD-6TM & $\mathrm{Y}$ & 4gap \\
\hline & Bm005226 & 531 & $2826: 44749-62984$ & - & 12 & NBD-5TM & $\mathrm{Y}$ & 4gap \\
\hline & $\mathrm{Bm} 000472$ & 590 & $1681: 5666445-5676633$ & + & 22 & NBD-4TM & $\mathrm{N}$ & \\
\hline & $\mathrm{Bm} 002712$ & 312 & $2529: 4492836-4497700$ & + & 5 & NBD & $\mathrm{N}$ & 1gap \\
\hline \multirow{3}{*}{$\mathrm{H}$} & Bm30646 & 689 & $2825: 75958-123240$ & & 12 & NBD-6TM & Y & \\
\hline & $\mathrm{Bm} 010726$ & 791 & $3003: 3211528-3248514$ & - & 26 & NBD-6TM & Y & lgap \\
\hline & $\mathrm{Bm} 010825$ & 176 & 3003:3337089-3344499 & + & 26 & NBD & $\mathrm{Y}$ & \\
\hline
\end{tabular}

The Silkworm protein name was written as the last six digits of the names in the form Bmxxxxxx (BGIBMGA012789:Bm012789).

\section{Result}

\subsection{ATP-binding Cassette Transporter Subfamily A $(A B C A)$}

ABCA subfamily proteins can be divided into full transporters and half transporters by distinctive conserved traits, two $\mathrm{ABCA}$ full transporters and five $\mathrm{ABCA}$ half transporters were found in the silkworm genome (Table 1). The two full transporters have a large extracellular loop between the first two transmembrane helices of each TMD, and a family specific motif located C-terminal of each NBF [29]. An analysis of the evolutionary relationship of these transporters to human, worm, and fruit fly ABCA proteins is shown in Fig.1. Bm012789 forms a subgroup with the hABCA5 cluster (hABCA5, 6, 8, 9, and 10). In mammals, murine ABCA5 protein was detected in lysosomes and late endosomes, ABCA5-/-mice developed characteristic symptoms of lysosomal diseases in ABCA5 expressing tissues (mainly heart and thyroid gland) and died when reaching adulthood [30]. Therefore, ABCA5 is proposed to play an important role in intracellular trafficking. The hABCA6 gene is highly expressed in liver, lung, heart, brain, and ovaries, which has been described to be up-regulated during macrophage differentiation and to be responsive to cholesterol concentrations $[31,32]$. Therefore, it is likely that ABCA6 plays an important role in macrophage lipid transport. Bm004188 forms a clade with CG31731 and Abt2. Bm009503 group together with ABCA12,ABCA13, and CG1819.The ABCA12 protein was localized in the lamellar granules of keratinocytes [33], where it may play a major role in the regulation of lipid trafficking. Moreover, the defect in cultured 
keratinocytes could be corrected after gene transfer, implying the possibility of a future gene therapy for harlequin ichthyosis. Human $\mathrm{ABCA} 13$ is the largest $\mathrm{ABC}$ transporter protein described to date, with a length of 5,058 amino acids and a predicted molecular weight of $>450 \mathrm{kDa}$ [34]. Elevated expression of the ABCA13 gene was found in leukemia, prostate tumor, and CNS tumor cell lines, where it could play a role in transport of xenobiotics and subsequent drug resistance. Bm007221 may be a putative orthologue of CG1718 and CG6052. Bm007217, Bm004187 and Bm007218 group together with Abt6.However, function of those proteins are uncertain, more research is needed to unravel the physiological significance of gene function in organisms. In mammals, ABCA proteins share a functional relation to lipid trafficking, individual transporters in the subfamily have adopted highly specialized roles in phosphor- and sphingolipid export machineries [35]. In consequence, it is not possible to assign specific putative roles to the silkworm ABCA transporters, though it appears likely that they are involved in lipid and xenobiotics trafficking processes.

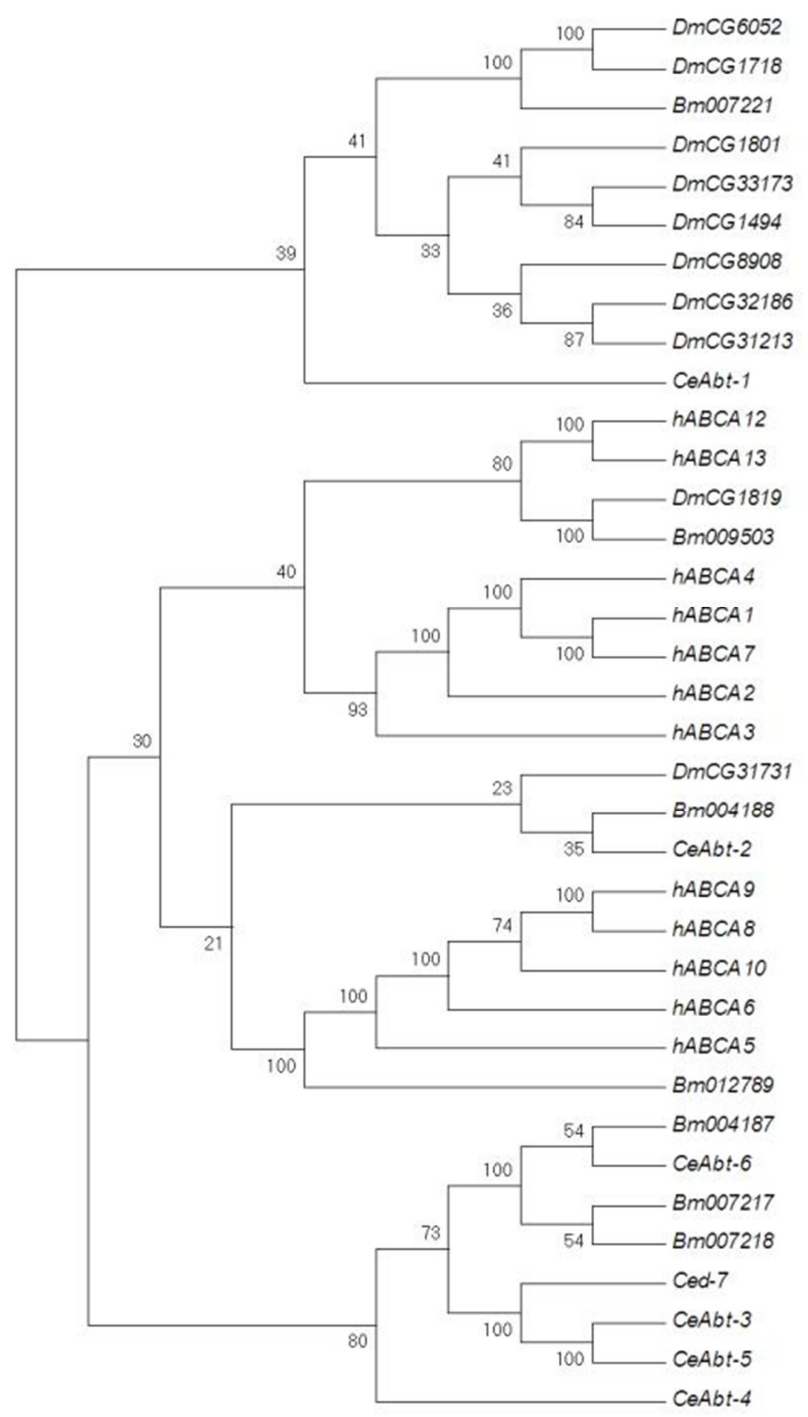

Figure 1. Phylogenetic tree of ABCA proteins in four eukaryotic genomes
Predicted amino acid sequences were aligned using ClustalX, and the alignment was used to generate a phylogenetic tree using the phylip 3.67, with the neighbour joining method. Bootstrapping was used to determine the relative support of the various branches (2,000 replicates, support expressed as percent). Bm, Bombyx mori, Dm Drosophila melanogaster; Ce Caenorhabditis elegans, h Homo sapiens.

\subsection{ATP-binding Cassette Transporter Subfamily B ( $A B C B)$}

The ABCB family can be divided into a group of full transporters that includes the drug efflux pump hABCB1/MDR1, and a group of half transporters. Five $\mathrm{ABCB}$ full transporters and four $\mathrm{ABCB}$ half transporters were found in the silkworm genome (Table 1). The evolutionary analysis of ABCB full transporters assigned the worm, human, and arthropod (combined Drosophila and bombyx) transporters into clearly distinguished clades (Fig.2), suggesting that this subfamily has diversified through lineage-specific gene duplications. One functional site of the human $\mathrm{ABCB} 1$ protein is the blood-brain barrier. Serendipitously, mice with the ABCB1 gene homologue "knocked-out" were observed to be extremely sensitive to an insecticide used in the animal care facility [36]. This observation provokes the question of a potential role of $\mathrm{ABCB}$ transporters in protecting insects from insecticides. Early reports have noted the similarity in sequence of three Drosophila ABCB full transporters and hABCB1/MDR1, and named these genes MDR49, MDR50, and MDR [37, 38]. While no further information is available on MDR50, the Drosophila gene most closely related to the five silkworm $\mathrm{ABCB}$ full transporters, $\mathrm{Bm} 011228, \mathrm{Bm} 000725$ and $\mathrm{Bm} 000724$ form a clade, and $\mathrm{Bm} 000724$ and $\mathrm{Bm} 000725$ are neighbouring genes showing a head-to-tail orientation and display $74 \%$ amino acid identity, suggesting they are the result of a tandem duplication. Silkworm Bm009452 and Bm007494 are closely related to the Drosophila MDR49 and MDR50 protein respectively, but easily recognizable homologs of the Drosophila MDR65 genes were not detected. A number of studies suggests a role of MDR49 and to a lesser extent also mdr65 in the biochemical defense against toxicants. A published study examined a Drosophila strain with a deletion in the MDR49 gene and showed that the mutant strain has an increased sensitivity to colchicines, but follow-up research has not been reported. A genetic polymorphism related to amatin resistance in Drosophila was mapped to the region of the MDR65 gene [39]. Colchicine exposure and heat shock increases the expression of mdr49, but not mdr65, in Drosophila larvae, while both genes were induced in tumors [40]. MDR49 was further found to be induced by polycyclic aromatic hydrocarbons, and shown to be involved in the transport of these chemicals [41]. 


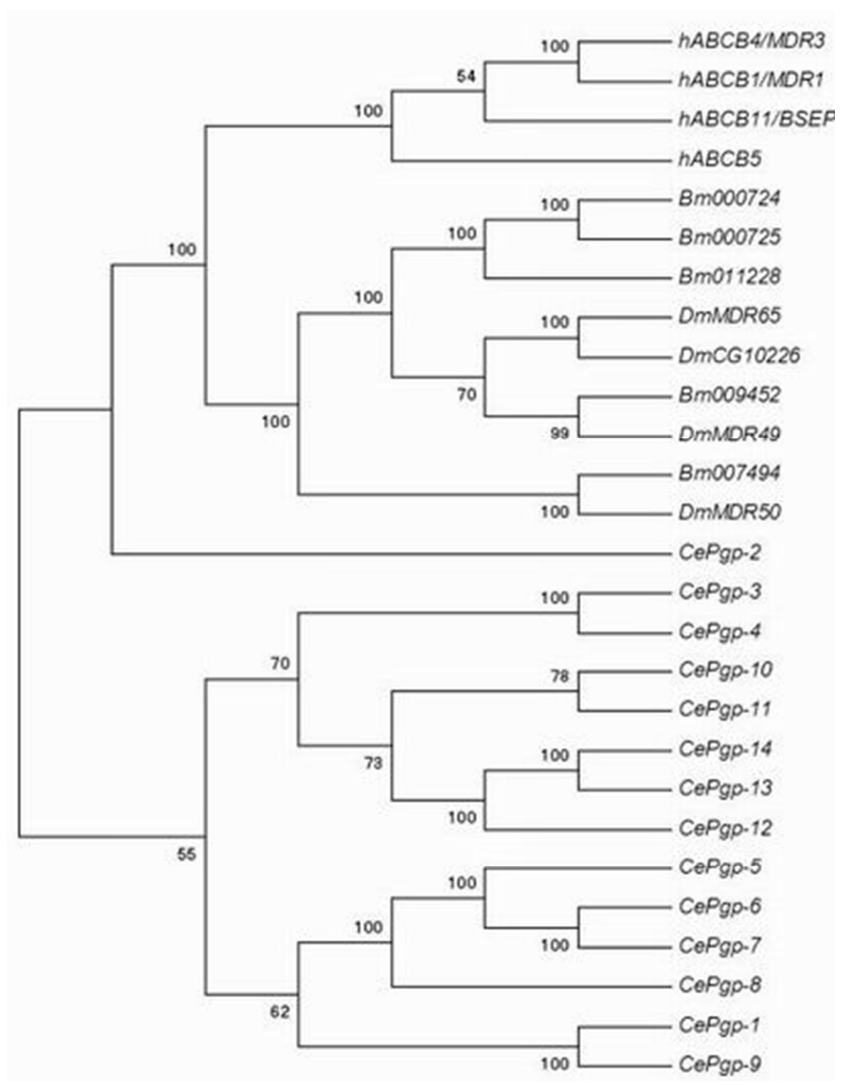

Figure 2. Phylogenetic tree of $A B C B$ subfamily full transporters in four eukaryotic genomes, derived according to the procedure outlined in the legend to Fig 1.

The phylogenetic analysis of $\mathrm{ABCB}$ half transporter revealed comparatively clear orthologue relations (Fig.3). Human mitochondrial transporters hABCB6, hABCB7, $\mathrm{hABCB} 8 / \mathrm{MABC} 1$ and $\mathrm{hABCB} 10 / \mathrm{MABC} 2$ function in iron metabolism and transport of $\mathrm{Fe} / \mathrm{S}$ protein precursors. The hABCB6, hABCB7, hABCB8/MABC1, and $\mathrm{hABCB} 10 / \mathrm{MABC} 2$ proteins each have one orthologue in silkworm (Bm005473, Bm00012743, Bm004142, and Bm008523, respectively). The human transporter associated with antigen processing (TAP) is a heterodimer of two ABCB proteins,hABCB2/TAP1 and ABCB3/TAP2.TAP translocates peptides derived from proteasomal degradation from the cytosol to the lumen of the endoplasmic reticulum, where their loading onto major histocompatibility complex (MHC) class I molecules occurs [42]. The function of hABCB9/TAPL (TAP-like) is currently unknown and its subcellular localisation is still under discussion [43]. It has been shown that TAPL is present in the lysosomal compartment [44], and it has been recently suggested that it might be involved in peptide presentation to MHC class II in dendritic cells [45]. As invertebrates lack the mammalian adaptive immune response, the lack of TAP/TAPL homologues in silkworm and Drosophila (Fig.3) is not unexpected. However, the presence of proteins orthologous to TAP/TAPL in C.elegans (Fig.3) suggests that these protein transporters might have further roles unrelated to antigen presentation.

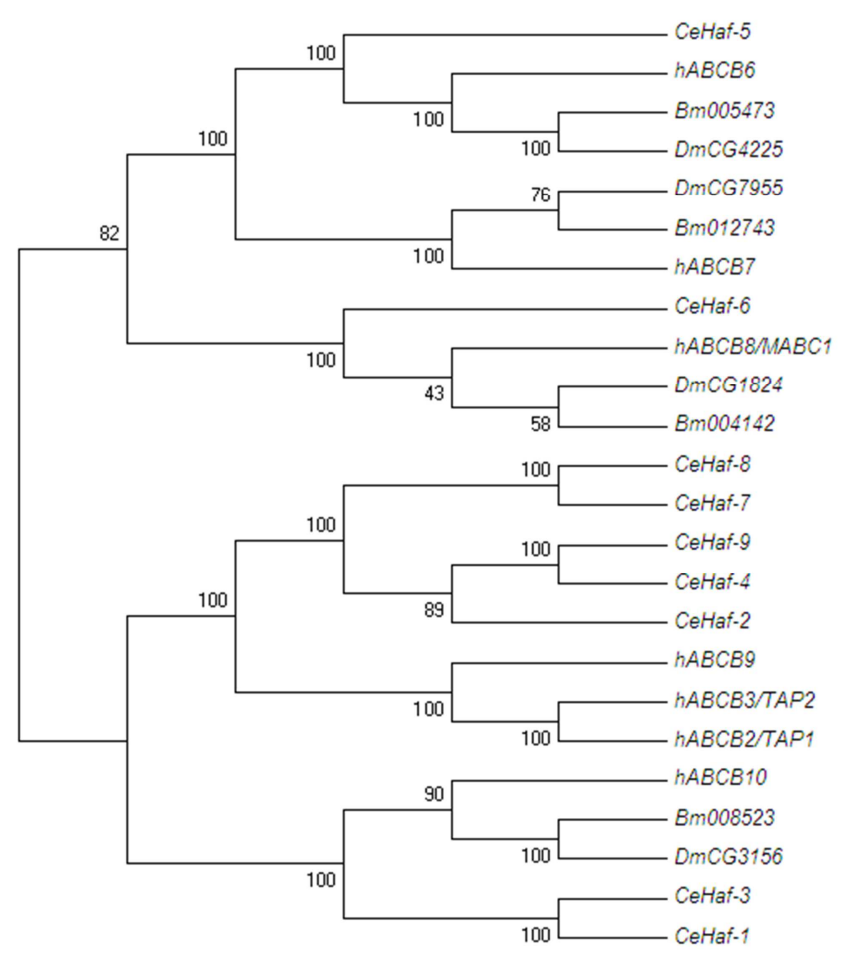

Figure 3. Phylogenetic tree of $A B C B$ subfamily half transporters in four eukaryotic genomes, derived according to the procedure outlined in the legend to Fig 1.

\subsection{ATP-binding Cassette Transporter Subfamily C (ABCC)}

The ABCC subfamily is functionally diverse, comprising the membrane-bound receptors SURs (sulfonylurea receptors), the chloride channel CFTR (cystic fibrosis transmembrane conductance regulator), and broad-specificity transporters called multidrug resistance-associated proteins (MRPs) that translocate a range of substrates including drugs, endogenous compounds and their glutathione and glucuronyl conjugates, glutathione, and cyclic nucleotides [46-48]. ABCC subfamily proteins are full transporters showing two TMDs and two NBDs. Within the human ABCC family, hABCC8/SUR1, hABCC9/SUR2 and certain MRPs called 'long' MRPs are unique in that they possess an additional N-terminal TMD called TMD0, which is lacking in ABCC7/CFTR and the remaining 'short' MRPs [49, 50]. Searches of the silkworm genome identified fifteen ABCC transporters, which include 6 full transporters and 9 half transporters (Table 1), and which further show a high number of loci lacking EST support (4 of 15 ABCC proteins Table 1). In contrast to the other subfamilies, $\mathrm{ABCC}$ has the most members of $\mathrm{ABC}$ transporter proteins. An analysis of the evolutionary relationship of these transporters to silkworm, human, worm, and fruit fly ABCC proteins is shown in Fig.4. Bm006882 is found in the same, well supported clade as human and Drosophila SURs, suggesting it is a SUR homologue. Indeed, Bm006882 displays a general architecture of SUR and 'long' MRPs, possessing the additional N-terminal TMD0. Together with previous reports of SUR-typical functional traits of the Drosophila protein CG5772 (SUR) [51, 52], the data strongly 
suggests that Bm006882 is indeed a SUR. In our phylogenetic analysis $\mathrm{Bm} 010330$ and $\mathrm{Bm} 010332$ group together with Drosophila CG6214 (Fig 4), which is a long MRP resembling hABCC1/MRP1 but awaiting in-depth functional characterisation [53, 54], and they predicted amino acid identities to CG6214 of $49.3 \%$ and $50.6 \%$, respectively. Bm003359 lacks of clear orthologous protein in Drosophila that makes the inference of function uncertain.Bm007785 groups together with Drosophila CG5789, but the protein physiological function is at present poorly known. According to the tree obtained in this study, Bm010636 is a putative orthologue of hABCC10 /MRP7 and Drosophila CG7806 (Fig. 4). hABCC10/MRP7 is capable of conferring a hABCC1/MRP1-type multidrug resistance phenotype in cellular models, but its physiological function is at present poorly understood [55]. Functional data are lacking on CG7806. Bm011220 and Bm010849 form a clade, it is uncertain that $\mathrm{Bm} 010849$ gene located in which chromosome in the silkworm genome.Bm007738 and six further silkworm proteins form a clade (Fig.4) that consists of a cluster of six neighbouring genes on scaffold 2888 (Table1). We speculate that the high number of $\mathrm{ABCC}$ genes in silkworm is due to extensive lineage specific gene duplications. It is known that the genomes of flies and worms contain a large number of duplicated genes, with a greater number of tandem or locally duplicated genes in the C. elegans than the Drosophila genome [56]. A large number of C. elegans annotated genes might be pseudogenes [57, 58]. A similar situation may exist in silkworm, at least with respect to the ABCC family, which contains a cluster of putative genes located in the same scaffold. Together these data suggest some silkworm MRPs could represent potential biochemical factors in the defense against toxicants; however, in the absence of functional data and in the view of the complex phylogeny of the ABCC subfamily this remains at present speculative.

\subsection{ATP-binding Cassette Transporter Subfamily D (ABCD)}

The ABCD subfamily contains half transporters located to the peroxisome that are involved in the import of fatty acids and/or fatty acyl-CoAs into this organelle [59]. The simultaneous posttranscriptional silencing of three C. elegans $\mathrm{ABCD}$ transporters disrupted offspring production in a previous study, suggesting developmental roles of peroxisomal ABC transporters [60]. Mutations in the $\mathrm{hABCD} 1 / \mathrm{ALDP}$ gene are the principal inherited defect in adrenoleukodystrophy, a clinically heterogeneous X-linked recessive disorder characterised by adrenal insufficiency and neuronal demyelination [61]. This study identified two ABCD transporters in the silkworm genome, Bm004616, and Bm012688 (Table1). The phylogenetic analysis shown that the high degree of conservation (amino acid identity of silkworm ABCD proteins to the closest Drosophila homologue between $54.8 \%$ and $68.5 \%$, and human homologue between $48.3 \%$ and $63.5 \%$ ), By some analysis of bioinformatic softwares, Bm004616 is not a imperfect transporter protein, which lacking transmembrane domains.

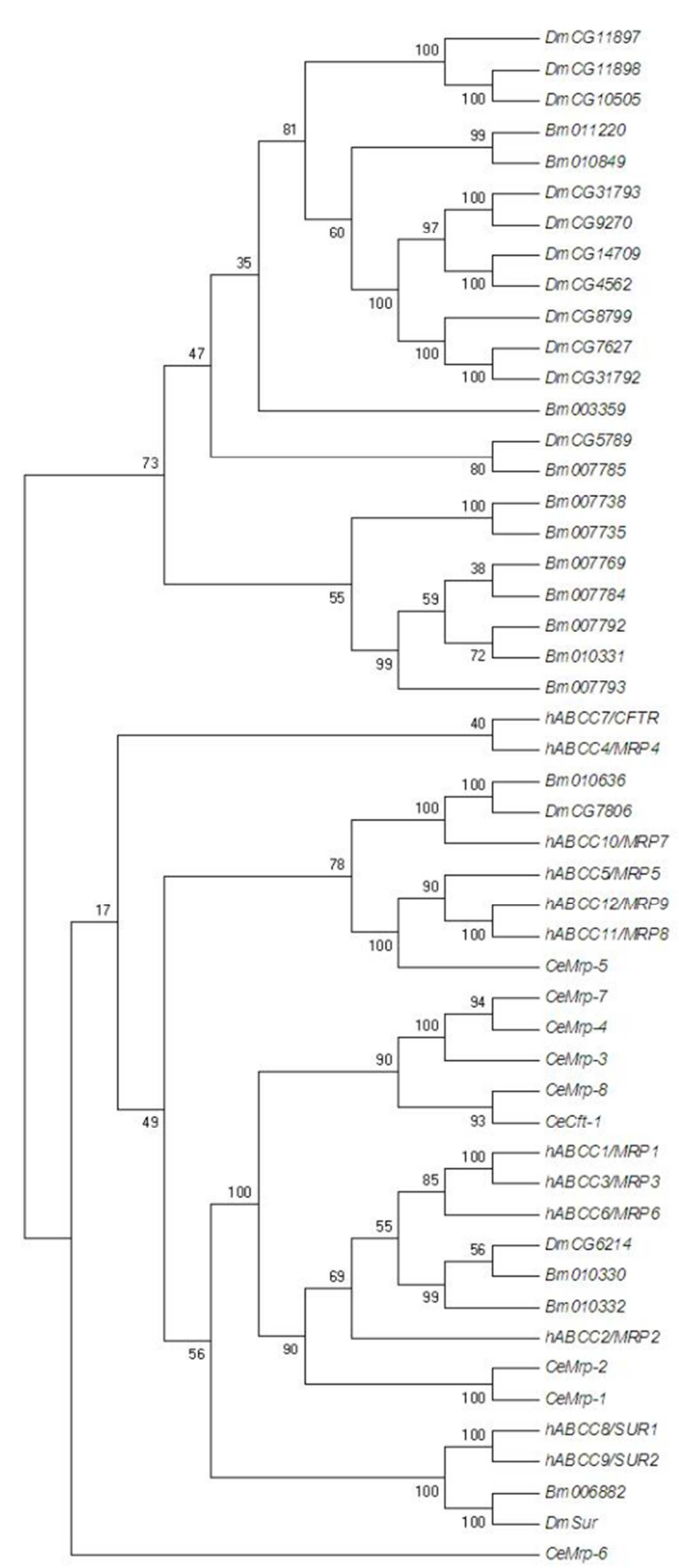

Figure 4. Phylogenetci tree of ABCC proteins in four eukaryotic genomes, derived according to the procedure outlined in the legend to Fig 1.

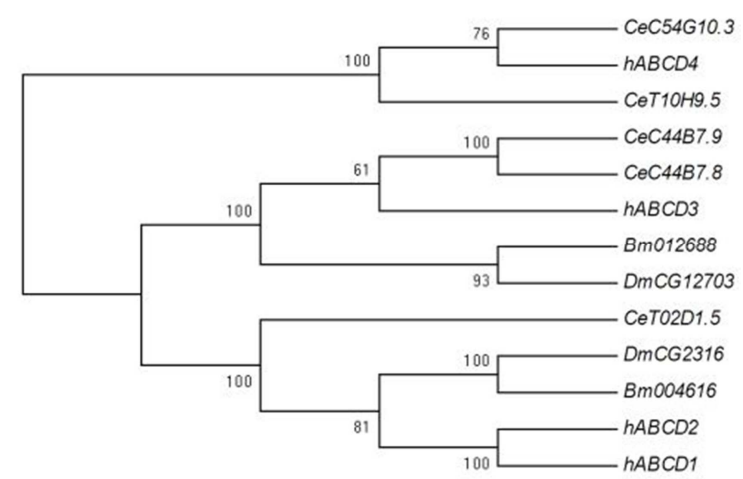

Figure 5. Phylogenetic tree of ABCD proteins in four eukaryotic genomes, derived according to the procedure outlined in the legend to Fig 1. 


\subsection{ATP-binding Cassette Transporter Subfamily $E$ and $F$ $(A B C E$ and $A B C F)$}

In yeast and apparently in humans, the $\mathrm{ABCE}$ and $\mathrm{F}$ subgroup $\mathrm{ABC}$-binding proteins are not transporters and contain only a pair of linked nucleotide binding domains and lack transmembrane domains [62]. The ABCE product is the oligo-adenylate binding protein that recognizes the poly-adenylate synthesized in eukaryotic cells in response to infections by some RNA viruses [63]. These genes are only present in multi-cellular organisms and probably have a role in the cellular stress response to certain viral infections. Most eukaryotes possess one ABCE protein, and silkworm conforms to this rule (Bm010129, Table1). Bm010129 groups together with CG5651 and hABCE1, and the predicted amino acid identity to CG1703 and hABCF1 of $86.8 \%$ and $76 \%$, respectively.Human ABCE1/RnaseLI was initially identified as an inhibitor of RnaseL [64]. Recent data indicate that human and yeast $\mathrm{ABCE}$ proteins have further a central role in translation initiation [65]. The ABCF product seemingly can activate the RNA-dependent protein kinase (PKR), an interferon-induced, RNA-activated enzyme that phosphorylates and inhibits the function of the translation initiation factor eIF-2 [4, 66, 67]. Three ABCF proteins have been identified in silkworm (Table 1). The phylogenetic analysis of $\mathrm{ABCE}$ and $\mathrm{ABCF}$ proteins was carried out together (Fig.6). As expected, Bm010129 fell into the clade containing other $\mathrm{ABCE}$ proteins. $\mathrm{ABCF}$ proteins were divided into three clades, and every clade contained one of the human, Drosophila, Caenorhabditis, and silkworm ABCF proteins (Fig. 6). Bm007869 groups together with CG1703 and hABCF1, and the predicted amino acid identity to CG1703 and hABCF1 of $61.1 \%$ and $52 \%$, respectively, $\mathrm{Bm} 007869$ is homologues to hABCF 1 , while Bm002004 and Bm006964 are homologues to hABCF2 and hABCF3, respectively.

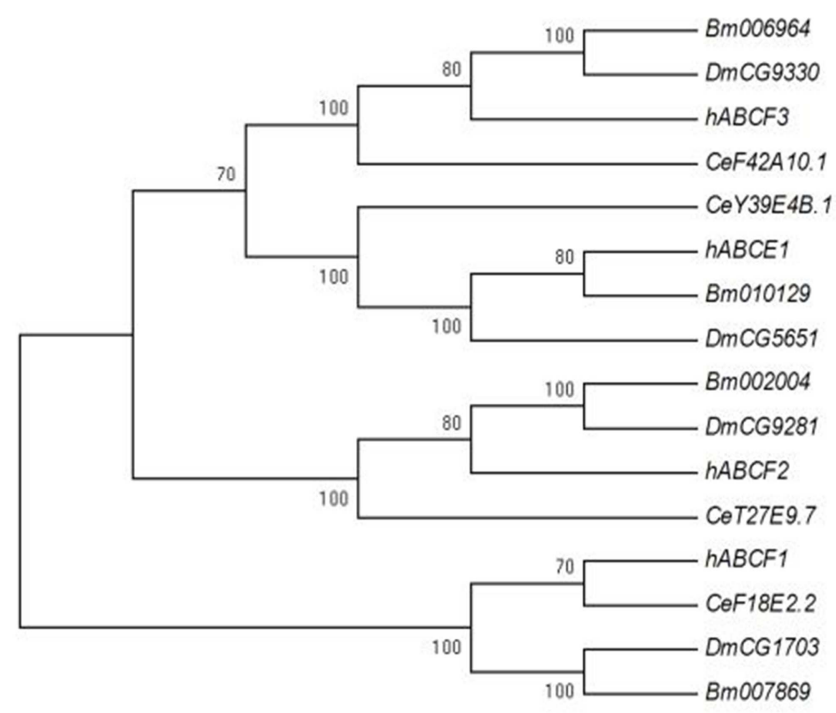

Figure 6. Phylogenetic tree of $A B C E$ and $A B C F$ proteins in four eukaryotic genomes, derived according to the procedure outlined in the legend to Fig 1 .

\subsection{ATP-binding Cassette Transporter Subfamily $G$ ( $A B C G)$}

The $G$ subfamily of ABC transporters consists of half-transporters, which need to form homo-or heterodimers to form a functional pump. In contrast to other $\mathrm{ABC}$ half transporters, ABCG proteins show reverse domain architecture, with the TMD being located C-terminally of the NBD. The human ABCG1 gene was originally called "human white" as it was cloned by degenerate PCR using primers based on the genomic sequence of the Drosophila white gene [68]. In mammals, ABCG1/WHITE1 and ABCG4 are involved in cellular cholesterol efflux to high density lipoprotein, ABCG5 and ABCG8 are associated with sitosterolemia characterized by accumulation of phytoand shellfish sterols. Unlike other members, ABCG2 (also termed the breast cancer resistance protein) is not involved in cholesterol efflux, but it exhibits broad substrate specificity to xenobiotic compounds. ABCG2 has been identified as a multidrug transporter that confers resistance on tumor cells. Evidence will be summarized suggesting that ABCG2 can also mediate the binding/transport of non-drug substrates, including free and conjugated steroids. Among the members, ABCG1 and ABCG4 exhibit high identity (70\%) at the amino acid level, whereas others exhibit low identity (at most $30 \%$ ). They are mammalian homologs of the Drosophila gene White, which forms heterodimers with scarlet or brown and plays an essential role in the cellular uptake of precursors of the eye pigments $[67,70,71]$. In yeast and in plants, certain ABCG subfamily proteins are reverse full transporters and called PDR (pleiotropic drug resistance) proteins [72]. In the phylogenetic analysis of ABCG proteins from the silkworm and other genomes, Bm012035 is a homologue of the Drosophila protein CG32091, and the protein of unknown function, an ABCG transporter believed to regulate intracellular ecdysone concentrations during development.

Phylogenetic analyses grouped Bm000220 and Bm000472 together in one clade with hABCG5 and 8, and two Drosophila proteins of uncertain function. Bm002712 dose not group together with Drosophila protein, and the inference function of the protein is unknown. Among the Drosophila eye pigment transporters, extensive characterization of Drosophila white mutants has shown that white forms a heterodimer with either brown or scarlet, two other ABCG family members. In the fly eye, these heterodimers localize to intracellular membranes of pigment granules that are present in specialized pigment cells. These pigment granules are considered to be modified lysosomes that form a specialized intracellular compartment. The two heterodimers, (white:brown and white:scarlet), are thought to transport different pigment precursors into these lysosome-like vesicles for the synthesis of ommochromes and pteridines, which together confer the "red eye" color [73]. Bm002922 (Bmwh3) is a homologue of the Drosophila white protein, and it is responsible for the transportation of ommochrome precursors and uric acid into pigment granules and urate granules, respectively[74], while brown and scarlet protein do not have a silkworm orthologue. 
The current first draft of the genome sequence contains a gap in some genes due to an assembly problem, and some proteins which have only a part of nucleotide binding domain are not appeared in the study. Bm30646 groups together with Drosophila proteins CG3164 and atet (ABC transporter expressed in trachea,[75]) as well as hABCG1 and 4, our laboratory has studied Bm30646 genes in detail, including sequencing the genomic region and verifying gene predictions by cDNA and PCR analysis (manuscript in preparation). Bm30646 gene was divided into two genes in genome sequence (unpublished data), assuming that these proteins might adopt roles in eye pigment transport in silkworm appears a reasonable hypothesis. However, it is noteworthy that recent evidence in Drosophila suggests additional neurobiological functions for the white protein $[76,77]$.

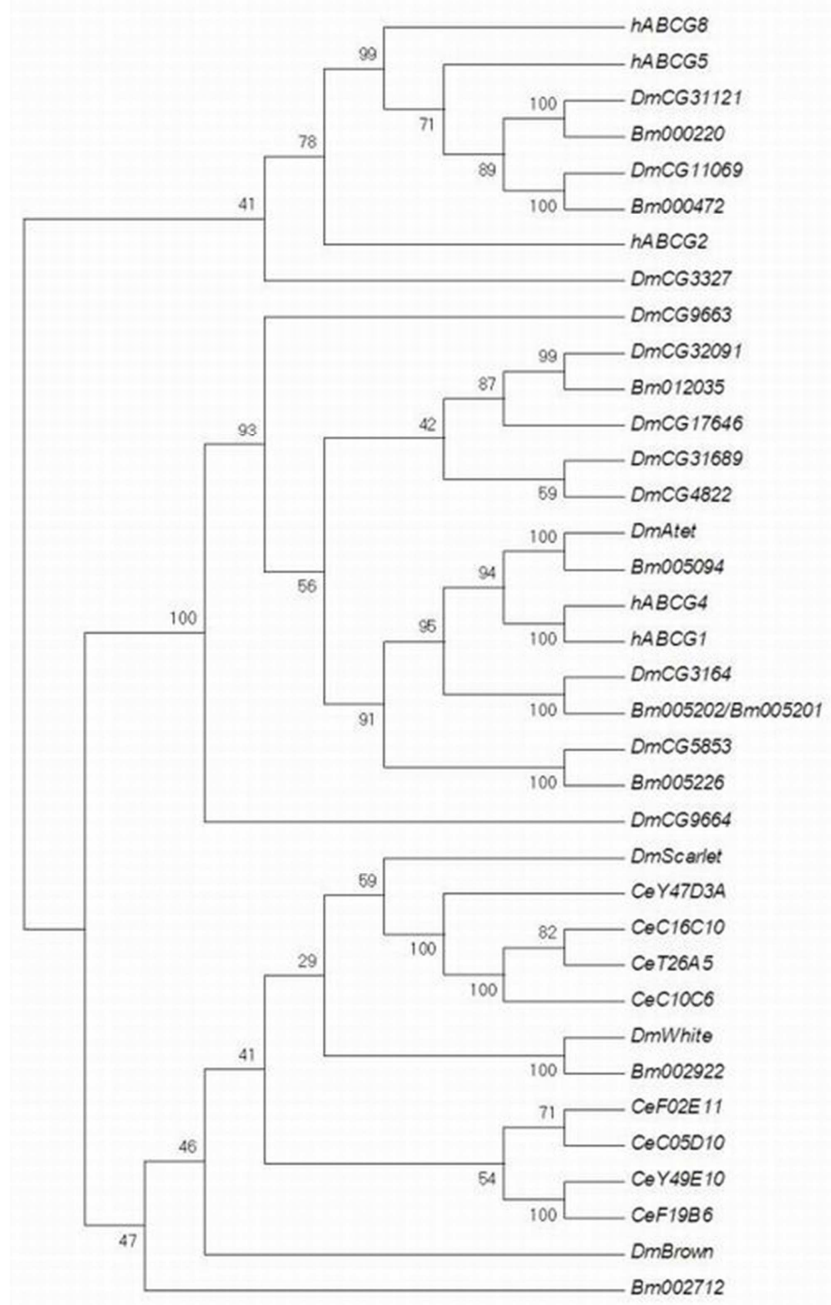

Figure 7. Phylogenetic tree of ABCG proteins in four eukaryotic genomes, derived according to the procedure outlined in the legend to Fig 1.

\subsection{ATP-binding Cassette Transporter Subfamily H (ABCH)}

The ABCH subfamily is lacking members in mammals, and has been identified for the first time in Drosophila [4]. At present, teleost fish are the only vertebrates known to possess $\mathrm{ABCH}$ transporters $[9,10] . \mathrm{ABCH}$ proteins are inverse half-transporters showing the same domain architecture as the members of the ABCG subfamily. The function of $\mathrm{ABCH}$ proteins is yet unknown. In silkworm, this subfamily is with two members the first least ABC subfamily (Table 1). The phylogenetic analysis assigned silkworm and Drosophila proteins in distinct clades (Fig. 8).

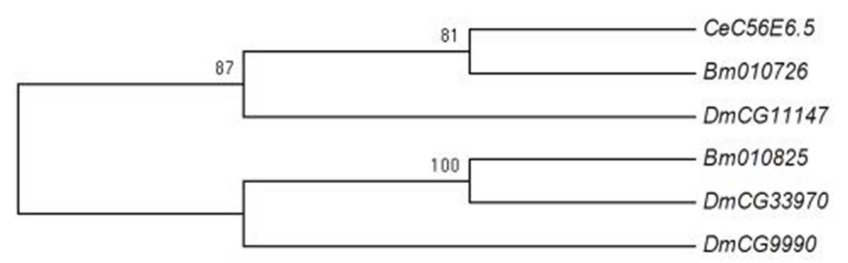

Figure 8. Phylogenetic tree of ABCH proteins in four eukaryotic genomes, derived according to the procedure outlined in the legend to Fig 1.

\section{Conclusion}

Genome-wide identification and characterization of the $\mathrm{ABC}$ transporters from the silkworm was conducted using bioinformatics methods. $47 \mathrm{ABC}$ transporter genes were identified in the silkworm genome which possesses members of all current $\mathrm{ABC}$ subfamilies A to $\mathrm{H}$. Each subfamily of $\mathrm{ABC}$ transporters of silkworm were compared to those from worm, fruit fly and human, and some interesting features between these four organisms were revealed.

A high conservation of silkworm $\mathrm{ABC}$ transporters were observed for proteins involved in fundamental cellular processes, including the half transporters of the ABCB subfamily, which function in iron metabolism and transport of $\mathrm{Fe} / \mathrm{S}$ protein precursors, and the members of subfamilies $\mathrm{ABCD}, \mathrm{ABCE}$ and $\mathrm{ABCF}$, which have roles in very long chain fatty acid transport. Comparison of the $\mathrm{ABC}$ transporters shows similarity between silkworm and Drosophila in that both lack homologue to the human proteins TAP, the protein translocator involved in antigen processing, and CFTR, a chloride channel regulated by ATP. The presence of proteins in silkworm that resemble the $\mathrm{ABC}$ drug efflux transporters $\mathrm{hABCB} 1 / \mathrm{MDR} 1$ and $\mathrm{hABCC} 1 / \mathrm{MRP} 1$ is in accordance with the hypothesis that these silkworm proteins might adopt roles in the biochemical defense against toxicants. Both $\mathrm{ABCE}$ and $\mathrm{F}$ gene products may be involved in an innate immune response to viral infections. As in the fly, $\mathrm{ABCH}$ transporters in silkworm are inverse half-transporters showing the same domain architecture as the members of the ABCG subfamily, and $\mathrm{ABCG}$ transporters may involve in transportation of ommochrome precursors and uric acid into pigment granules and urate granules.

These results might provide new insights for further study on the function of $\mathrm{ABC}$ transporters in the silkworm genome.

\section{Acknowledgements}

This work was supported by the National Natural Science Foundation of China (No. 31272508). 


\section{References}

[1] Holland IB. ABC transporters, mechanisms and biology: an overview. Essays Biochem. 2011 Sep 7; 50(1): 1-17.

[2] Wilkens S. Structure and mechanism of $\mathrm{ABC}$ transporters. F1000Prime Rep. 2015 Feb 3; 7: 14.

[3] Dassa E, Bouige P. The $\mathrm{ABC}$ of $\mathrm{ABCs}$ : a phylogenetic and functional classification of $\mathrm{ABC}$ systems in living organisms. Res Microbiol 2001, 152: 211-229.

[4] Dean M, Hamon Y, Chimini G. The human ATP-binding cassette (ABC) transporter superfamily. J Lipid Res 2001, 42: 1007-1017.

[5] Hollenstein K, Frei DC, Locher KP. Structure of an ABC transporter in complex with its binding protein. Nature 2007, 446: 213-216.

[6] Dawson RJ, Locher KP. Structure of a bacterial multidrug ABC transporter. Nature 2006, 443: 180-185.

[7] Klein I, Sarkadi B, Váradi A. An inventory of the human ABC proteins. Biochim Biophys Acta 1999, 1461: 237-262.

[8] Dean M., Rzhetsky A, Allikmets R. The human ATP-binding cassette (ABC) transporter superfamily. Genome Res 2001, 11: $1156-1166$.

[9] Annilo T, Chen ZQ, Shulenin S, et al. Evolution of the vertebrate $\mathrm{ABC}$ gene family: analysis of gene birth and death. Genomics 2006, 88: 1-11.

[10] Dean M, Annilo T. Evolution of the ATP-binding cassette $(\mathrm{ABC})$ transporter superfamily in vertebrates. Annu Rev Genomics Hum Genet 2005, 6: 123-142.

[11] $\mathrm{Wu} \mathrm{ZB}, \mathrm{Wu}$ JM. The G subfamily of ATP-binding cassette $(\mathrm{ABC})$ transporters and human diseases. Journal of Jiangsu University (Medicine Edition) 2008, 18(3): 861-866.

[12] Gottesman MM, Ambudkar SV. Overview: ABC transporters and human disease. Bioenerg Biomembr 2001, 33: 453-458.

[13] Li G, Gu HM, Zhang DW. ATP-binding cassette transporters and cholesterol translocation. IUBMB Life. 2013 Jun; 65(6): 505-12.

[14] Hlaváč V, Souček P. Role of family D ATP-binding cassette transporters (ABCD) in cancer. Biochem Soc Trans. 2015 Oct 1; 43(5): 937-42.

[15] Ween MP, Armstrong MA, Oehler MK, Ricciardelli C. The role of $\mathrm{ABC}$ transporters in ovarian cancer progression and chemoresistance. Crit Rev Oncol Hematol. 2015 Nov; 96(2): 220-56.

[16] Gatti L, Cossa G, Beretta GL, Zaffaroni N, Perego P. Novel insights into targeting ATP-binding cassette transporters for antitumor therapy. Curr Med Chem. 2011; 18(27): 4237-49.

[17] Bloise E, Ortiga-Carvalho TM, Reis FM, Lye SJ, Gibb W, Matthews SG. ATP-binding cassette transporters in reproduction: a new frontier. Hum Reprod Update. 2015 Nov 5. pii: dmv049. [Epub ahead of print]

[18] Ling, V, Kartner, N, Sudo, T, et al. Multidrug-resistance phenotype in Chinese hamster ovary cells. Cancer Treat Rep $1983,67,869-874$.
[19] Gottesman, MM. Drug-resistant mutants: selection and dominance analysis. Methods Enzymol 1987, 151: 113-121.

[20] Riordan JR, Ling V. Purification of P-glycoprotein from plasma membrane vesicles of chinese hamster ovary cell mutants with reduced colchicine permeability. Biol Chem 1979, 254: 12701-12705.

[21] Gerlach JH, Endicott JA, Juranka PF, et al. Homology between P-glycoprotein and a bacterial heamolysin transport protein suggests a model for multidrug resistance. Nature 1986, 324 : 485-489.

[22] Cole SP, Bhardwaj G, Gerlach JH, et al. Overexpression of a transporter gene in a multidrug-resistant human lung cancer cell line. Science 1992, 258: 1650-1654.

[23] Doyle LA, Yang W, Abruzzo LV, et al. A multidrug resistance transporter from human MCF-7 breast cancer cells. Proc Natl Acad Sci USA 1998, 95: 15665-15670.

[24] Gottesman MM, Fojo T, Bates SE. Multidrug resistance in cancer: Role of ATP-dependent transporters. Nat Rev Cancer 2002, 2: 48-58.

[25] Bodo A, Bakos E, Szeri F, et al. The role of multid-drug transporters in drug availability, metabolism and toxicity. Toxicol Lett 2003, 140-141: 133-143.

[26] Xia et al. [http://silkworm.genomics.org.cn/].

[27] Kerr ID. Structure and association of ATP-binding cassette transporter nucleotide-binding domains. Biochim Biophys Acta 2002, 1561: 47-64.

[28] ExPASy (Expert Protein Analysis System) proteomics server of the Swiss Institute of Bioinformatics (SIB), Scan Prosite facility [http://expasy.org/tools/scanprosite/].

[29] Peelman F, Labeur C, Vanloo B, et al. Characterization of the ABCA transporter subfamily: identification of prokaryotic and eukaryotic members, phylogeny and topology. Mol Biol 2003, 325: 259-274.

[30] Kubo Y, Sekiya S, Ohigashi M, et al. ABCA5 resides in lysosomes, and ABCA5 knockout mice develop lysosomal disease-like symptoms. Mol Cell Biol 2005, 25: 4138-4149.

[31] Albrecht C, Viturro E.The ABCA subfamily gene and protein structures, functions and associated hereditary diseases $[\mathrm{J}]$. Pflugers Arch - Eur J Physiol 2007, 453: 581-589.

[32] Kaminski WE, Wenzel JJ, Piehler A, et al. ABCA6, a novel a subclass $\mathrm{ABC}$ transporter. Biochem Biophys Res Commun 2001, 285: 1295-1301.

[33] Akiyama M, Sugiyama-Nakagiri Y, Sakai K, et al. Mutations in lipid transporter $\mathrm{ABCA} 12$ in harlequin ichthyosis and functional recovery by corrective gene transfer. Clin Invest 2005, 115: 1777-1784.

[34] Prades C, Arnould I, Annilo T, et al. The human ATP binding cassette gene ABCA13, located on chromosome 7p12.3, encodes a 5058 amino acid protein with an extracellular domain encoded in part by a $4.8-\mathrm{kb}$ conserved exon. Cytogenet Genome Res 2002, 98: 160-168.

[35] Wenzel JJ, Piehler A, Kaminski WE. ABCA subclass proteins: gatekeepers of cellular phospho- and sphingolipid transport. Front Biosci 2007, 12: 3177-3193. 
[36] Schinkel AH, Mol CA, Wagenaar E, et al. Multidrug resistance and the role of P-glycoprotein knockout mice. Eur Cancer 1995, 31A, 1295-1298.

[37] $\mathrm{Wu} C T$, Budding M, Griffin MS, et al. Isolation and characterization of Drosophila multidrug resistance gene homologs. Mol Cell Biol 1991, 11: 3940-3948.

[38] Gerrard B, Stewart C, Dean M. Analysis of Mdr50: A Drosophila P-glycoprotein/multidrug resistance gene homolog. Genomics 1993, 17: 83-88.

[39] Begun DJ, Whitley P. Genetics of alpha-amanitin resistance in a natural population of Drosophila melanogaster. Heredity 2000, 85(Pt2): 184-190.

[40] Tapadia MG, Lakhotia SC. Expression of mdr49 and mdr65 multidrug resistance genes in larval tissues of Drosophila melanogaster under normal and stress conditions. Cell Stress Chaperones 2005, 10: 7-11.

[41] Vache C, Camares O, Cardoso-Ferreira MC, et al. A potential genomic biomarker for the detection of polycyclic aromatic hydrocarbon pollutants: multidrug resistance gene 49 in Drosophila melanogaster. Environ Toxicol Chem 2007, 26: 1418-1424.

[42] Abele R, Tampe R. Modulation of the antigen transport machinery TAP by friends and enemies. FEBS Lett 2006, 580: 1156-1163.

[43] Herget M, Tampe R. Intracellular peptide transporters in human compartmentalization of the "peptidome". Pflugers Arch 2007, 453: 591-600.

[44] Zhang F, Zhang W, Liu L, et al. Characterization of ABCB9, an ATP binding cassette protein associated with lysosomes. Biol Chem 2000, 275: 23287-23294.

[45] Sturm A, Cunningham P, Dean M. The ABC transporter gene family of Daphnia pulex. BMC Genomics 2009, 10: 170 .

[46] Aleksandrov AA, Aleksandrov LA, Riordan JR. CFTR (ABCC7) is a hydrolyzable-ligand-gated channel. Pflugers Arch 2007, 453: 693-702.

[47] Moreau C, Prost AL, Derand R, et al. SUR, ABC proteins targeted by KATP channel openers. Mol Cell Cardiol 2005, 38: 951-963.

[48] Kruh GD, Belinsky MG. The MRP family of drug efflux pumps [J].Oncogene 2003, 22: 7537-7552.

[49] Conti LR, Radeke CM, Shyng SL, et al. Transmembrane topology of the sulfonylurea receptor SUR1. Biol Chem 2001, 276: 41270-41278.

[50] Deeley RG, Westlake C, Cole SP. Transmembrane transport of endo- and xenobiotics by mammalian ATP-binding cassette multidrug resistance proteins. Physiol Rev 2006, 86: 849-899.

[51] Akasaka T,Klinedinst S, Ocorr K,et al. The ATP-sensitive potassium (KATP) channel-encoded dSUR gene is required for Drosophila heart function and is regulated by tinman. Proc Natl Acad Sci USA 2006, 103: 11999-12004.

[52] Nasonkin I, Alikasifoglu A, Ambros e C, et al.A novel sulfonylurea receptor amily member expressed in the embryonic Drosophila dorsal vessel and tracheal system. Biol Chem 1999, 274: 29420-29425.
[53] Tarnay JN, Szeri F, Ilias A, et al. The dMRP/CG6214 gene of Drosophila is evolutionarily and functionally related to the human multidrug resistance-associated protein family. Insect Mol Biol 2004, 13: 539-548.

[54] Grailles M, Brey PT, Roth CW. The Drosophila melanogaster multidrug-resistance protein 1 (MRP1) homolog has a novel gene structure containing two variable internal exons. Gene 2003, 307: 41-50.

[55] Kruh GD, Guo Y, Hopper-Borge E, et al. ABCC10, ABCC11, and ABCC12. Pflugers Arch 2007, 453: 675-684.

[56] Rubin GM, Yandell MD, Wortman JR, et al. Comparative genomics of the eukaryotes. Science 2000, 287: 2204-2215.

[57] Harrison PM, Echols N, Gerstein MB. Digging for dead genes: an analysis of the characteristics of the pseudogene population in the Caenorhabditis elegans genome. Nucleic Acids Res 2001, 29: $818-830$.

[58] Mounsey A, Bauer P, Hope IA.Evidence suggesting that a fifth of annotated Caenorhabditis elegans genes may be pseudogenes. Genome Res, 2002, 12: 770-775.

[59] Theodoulou FL, Holdsworth M, Baker A: Peroxisomal ABC transporters. FEBS Lett 2006, 580: 1139-1155.

[60] Petriv OI, Pilgrim DB, Rachubinski RA, et al. RNA interference of peroxisome-related genes in C. elegans: a new model for human peroxisomal disorders. Physiol Genomics 2002, 10: 79-91.

[61] Berger J, Gartner J. X-linked adrenoleukodystrophy: clinical, biochemical and pathogenetic aspects. Biochim Biophys Acta 2006, 1763: 1721-1732.

[62] Kerr ID. Sequence analysis of twin ATP binding cassette proteins involved in translational control, antibiotic resistance, and ribonuclease L inhibition. Biochem Biophys Res Commun 2004, 315: 166-173.

[63] Allikmets R, Schriml LM, Hutchinson A, et al. A human placenta-specific ATP-binding cassette gene (ABCP) on chromosome $4 \mathrm{q} 22$ that is involved in multidrug resistance. Cancer Res 1998, 58, 5337-5339.

[64] Zhou A, Hassel BA, Silverman RH. Expression cloning of 2-5Adependent RNAase: a uniquely regulated mediator of interferon action. Cell 1993, 72: 753-765.

[65] Chen ZQ, Dong J, Ishimura A, et al. The essential vertebrate ABCE1 protein interacts with eukaryotic initiation factors. Biol Chem 2006, 281: 7452-7457.

[66] Marton MJ, Vazquez de Aldana CR, Qiu H. Evidence that GCN1 and GCN20, translational regulators of GCN4, function on elongating ribosomes in activation of eIF2alpha kinase GCN2. Mol Cell Biol 1997, 17, 4474-4489.

[67] Tyzack JK, Wang X., Belsham GJ, et al. ABC50 interacts with eukaryotic initiation factor 2 and associates with the ribosome in an ATP-dependent manner. Biol Chem 2000, 275, 34131-34139.

[68] Savary S, Denizot F. Luciani M, et al. Molecular cloning of a mammalian $\mathrm{ABC}$ transporter homologous to Drosophila white gene, Mamm Genome 1996, 7: 673-676.

[69] Dreesen TD, Johnson DH, Henikoff S. The brown protein of Drosophila melanogaster is similar to the white protein and to components of active transport complexes. Mol Cell Biol 1988, 8: 5206-5215. 
[70] Sullivan DT, Bell LA, Paton DR, et al. Purine transport by malpighian tubules of pteridine-deficient eye color mutants of Drosophila melanogaster. Biochem Genet 1979, 17: 565-573.

[71] Tearle RG, Belote JM, McKeown M, et al. Cloning and characterization of the scarlet gene of Drosophila melanogaster. Genetics 1989, 122: 595-606.

[72] Crouzet J, Trombik T, Fraysse AS, et al. Organization and function of the plant pleiotropic drug resistance $\mathrm{ABC}$ transporter family. FEBS Lett 2006, 580: 1123-1130.

[73] Paul T. Tarra, Elizabeth J. Tarling, Dragana D. Bojanic, et al. Emerging new paradigms for $\mathrm{ABCG}$ transporters. Biochimica et Biophysica Acta 2009, 1791: 584-593

[74] Natuo Ko^moto, Guo-Xing Quan, Hideki Sezutsu, et al. A single-base deletion in an $\mathrm{ABC}$ transporter gene causes White eyes, White eggs, and translucent larval skin in the silkworm $w$ ${ }_{30 e}$ mutant. Insect Biochemistry and Molecular Biology 2009, 39: $152-156$.

[75] Kuwana H, Shimizu-Nishikawa K, Iwahana H, et al. Molecular cloning and characterization of the $\mathrm{ABC}$ transporter expressed in Trachea (ATET) gene from Drosophila melanogaster. Biochim Biophys Acta 1996, 1309: 47-52.

[76] Campbell JL, Nash HA. Volatile general anesthetics reveal a neurobiological role for the white and brown genes of Drosophila melanogaster. Neurobiol 2001, 49: 339-349.

[77] Lee HG, Kim YC, Dunning JS, et al. Recurring ethanol exposure induces disinhibited courtship in Drosophila. PLoS ONE 2008, 3: e1391. 